\title{
Initial Data for General Relativity with Toroidal Conformal Symmetry
}

\author{
R. Beig and S. Husa \\ Institut für Theoretische Physik, Universität Wien, Vienna, Austria
}

(November 12, 2018)

\begin{abstract}
A new class of time-symmetric solutions to the initial value constraints of vacuum General Relativity is introduced. These data are globally regular, asymptotically flat (with possibly several asymptotic ends) and in general have no isometries, but a $U(1) \times U(1)$ group of conformal isometries. After decomposing the Lichnerowicz conformal factor in a double Fourier series on the group orbits, the solutions are given in terms of a countable family of uncoupled ODEs on the orbit space.
\end{abstract}

Typeset using REVTEX 
If one casts General Relativity in canonical form one finds that the Einstein equations split into two parts. One part, the constraints, has no time derivatives in it: the equations are conditions on the initial data. The other part, the evolution equations, determine how this initial data develop in time. The constraints are a highly underdetermined system which can be turned into elliptic equations by a judicious choice of the free data.

The simplest version of the constraints occurs in the so-called moment-of-time-symmetry (or time-symmetric) problem, where the velocity part of the data, namely the extrinsic curvature of the initial 3-manifold $M^{\prime}$, is set to zero and the only remaining equation is $R[g]=0$, with $g$ a Riemannian metric on the Cauchy slice and $R$ the scalar curvature of $g$.

The standard approach, pioneered by Lichnerowicz, is to select a base metric $g$ and construct a solution metric $g^{\prime}$ by means of a conformal transformation $g^{\prime}=\psi^{4} g$. One then gets a linear elliptic PDE, namely

$$
L_{g} \psi:=\left(-\triangle_{g}+\frac{1}{8} R[g]\right) \psi=0, \quad \psi>0
$$

for the conformal factor. In the asymptotically flat case studied here one in addition assumes that $g^{\prime}$ tends to the flat metric at infinity. One then requires that $\psi$, in addition to obeying Eq. (1), go to one in that limit.

Simplification should occur if one imposes some symmetry on $g$. Completely reducing this 3 dimensional PDE problem to a one dimensional ODE by imposing a symmetry with 2 dimensional orbits does not really work. In the case of spherical symmetry this would, by the Birkhoff theorem, leave us with data for the Schwarzschild solution. In the cylindrically symmetric case, using the boundary condition, only Minkowski space remains [1]. Thus, in the standard approach, the simplest solution that has been identified is to assume that the free data is axially symmetric which reduces Eq. (1) to a two dimensional PDE, and much effort, especially by the numerical community, has been devoted to solving it.

In this paper we wish to introduce a new symmetry in the base metric which gives us a large class of non-trivial solutions while at the same time allowing us to benefit from the underlying linearity of the equation. With this symmetry we can reduce the equation 
to a set of uncoupled linear ODEs. This is a remarkable simplification which offers major benefits in a whole range of problems. The key idea is to use the freedom in choosing the base metric $g$ in a conformal equivalence class, particularly one can choose a base metric that lives on a compact manifold which can be opened up to an asymptotically flat one by conformal decompactification (analogous to stereographic projection).

The physical data we are considering are data defined on a 3-dimensional manifold $M^{\prime}$ which may have several ends and are asymptotically flat near each of them. They possess two commuting, orthogonal conformal Killing vector fields. Their action extends to a $U(1) \times U(1)$ action on the many-point compactification $S^{3}$ of $M^{\prime}$. After a suitable conformal rescaling of the metric, this action becomes in fact an isometry, and is thus of the "polarized Gowdy type" [2]. In the special case of just one point-at-infinity, which is a fixed point of one $U(1)$ factor, these data have this $U(1)$ factor as an isometry and are thus a subclass of Brill waves. The fact that the group of all conformal symmetries of the physical data extends to an isometry group only after conformal compactification turns out to be a blessing rather than a drawback.

Even in the absence of symmetry it is useful to replace Eq. (11), on $R^{3}$ say, together with the boundary condition $\psi \rightarrow 1$ at infinity, by the equation

$$
L_{g} G=4 \pi \delta_{\Lambda}, \quad G>0
$$

on the one-point compactification $S^{3}$ of $M^{\prime}$ with $g$ now being a Riemannian metric on $S^{3}$ and $\delta_{\Lambda}$ the Dirac delta function concentrated at some point $\Lambda \in S^{3}$. It is then easy to see, using the known asymptotic behaviour of $G$ near $\Lambda$, that the metric $g_{a b}^{\prime}=G^{4} g_{a b}$ on $R^{3} \cong S^{3} \backslash \Lambda$ is asymptotically flat near $\Lambda$ and satisfies $R\left[g^{\prime}\right]=0$ on account of Eq. (2)). A solution $G>0$ is known to exist, iff $\lambda_{1}$, the lowest eigenvalue of $L_{g}$, is positive [3]. In particular, this existence is insensitive to the choice of the point $\Lambda$ which we will use later when we construct solutions with many asymptotic ends.

To solve Eq. (2) on the compact manifold $S^{3}$ we subtract from $G$ a quantity $G_{l o c}$, taken to be a suitable approximation to the Hadamard fundamental solution 四 of $L_{g}$ near $\Lambda$, 
arbitrarily extended to all of $S^{3}$. By including a sufficient number of terms in $G_{l o c}$, we can arrange for the r.h.s. in

$$
L_{g} \phi=L_{g}\left(G-G_{l o c}\right)=\rho_{\Lambda}
$$

to be as smooth at $\Lambda$ as we please. Solving the regular equation (3) on $S^{3}$ numerically the usual problems arising from having to use a finite grid for an infinite region $\left(R^{3}\right.$ in this case) are avoided. Moreover, in the presence of a $U(1) \times U(1)$ isometry we can introduce adapted coordinates, which will allow us to reduce the PDE (3) to a system of uncoupled ODEs.

For the basic setup it is best to start by obtaining the standard metric $\stackrel{o}{a b}_{a b}$ on $S^{3}$ by inverse stereographic projection from $R^{3}$ with flat metric $\stackrel{o}{g}_{a b}^{\prime}$. Let $z, r, \varphi$ be standard cylindrical coordinates on $\left(R^{3}, \stackrel{o}{g}\right)$. Taking $\Omega=4\left(1+4\left(r^{2}+z^{2}\right)\right)^{-1}$, the metric $\stackrel{o}{g}_{a b}=\Omega^{2} \stackrel{o}{g}_{a b}^{\prime}$ extends to the standard one on $S^{3}$. Furthermore $\eta^{a}=\left(\frac{\partial}{\partial \varphi}\right)^{a}$ extends to a Killing vector on $\left(S^{3}, \stackrel{o}{g}\right.$ ab $)$. With $\rho^{2}=\eta^{a} \eta^{b} \stackrel{o}{g}_{a b}$ we have that $\eta^{a} \rho_{a}=0$ and

$$
\stackrel{o}{D}_{a} \stackrel{o}{\eta}_{b}=2 \rho^{-1} \rho_{[a} \stackrel{o}{\eta}_{b]}, \quad \rho_{a b}=\rho^{-3} \stackrel{o}{\eta}_{a} \stackrel{o}{\eta}_{b}-\rho \stackrel{o}{g}_{a b}
$$

where we use the definitions

$$
\stackrel{o}{D}_{c} \stackrel{o}{g}_{a b}=0, \quad \rho_{a}=\stackrel{o}{D}_{a} \rho, \quad \rho_{a b}=\stackrel{o}{D}_{a} \stackrel{o}{D}_{b} \rho .
$$

Next define

$$
\xi^{a}=\rho^{-1 \stackrel{o a b c}{\epsilon}} \rho_{b} \stackrel{o}{\eta}_{c}
$$

obeying $\xi^{a} \xi^{b} \stackrel{o}{a b}_{a b}=1-\rho^{2}=: \sigma^{2}, \xi^{a} \rho_{a}=0, \xi^{a} \eta^{b} \stackrel{o}{g}_{a b}=0$, and

$$
\stackrel{o}{D} \xi_{a}=2 \sigma^{-1} \sigma_{[a} \xi_{b]} .
$$

It thus follows from equations (4, 5, 6) that $(\xi, \eta)$ form a pair of commuting, orthogonal and hypersurface-orthogonal Killing vectors of $\stackrel{o}{g}_{a b}$ spanning $\rho=\rho_{0}=$ const. where $\rho_{0}$ ranges between 0 and 1 . For $\rho_{0} \neq 0,1$ these are flat tori of constant mean curvature changing sign at the Clifford torus for which $\rho_{0}=\frac{1}{\sqrt{2}}$. The sets $\rho=0(\rho=1)$ are closed lines on which 
$\eta^{a}\left(\xi^{a}\right)$ is zero (the "axes"). In fact, $\rho=0$ (respectively $\rho=1$ ) are linked great circles on $S^{3}$, corresponding to the $z$-axis (respectively the circle $z=0, r=1 / 2$ ) after stereographic projection. Using coordinates $(\rho, \chi, \varphi)$ where $\xi^{a}=\left(\frac{\partial}{\partial \chi}\right)^{a}$ and $\chi=$ const. are orthogonal to $\xi^{a}$ we find that $\chi$, like $\varphi$, has periodicity $2 \pi$. The coordinate $\chi$ can be fixed by requiring $\Lambda$, the point at infinity under stereographic projection, to be at $\rho=0, \chi=0$. The totally geodesic surfaces $\chi=$ const. $(\varphi=$ const. $)$ are metric hemispheres intersecting at $\rho=1$ $(\rho=0)$ with $\{\chi=\alpha\} \cup\{\chi=\alpha+\pi\}(\{\varphi=\beta\} \cup\{\varphi=\beta+\pi\})$ being smoothly embedded $S^{2}$ 's. Under stereographic projection the surfaces $\chi=$ const. do not change topology except for $\{\chi=0\} \cup\{\chi=\pi\}$ which gets decompactified into $\{z=0, r \geq 1 / 2\} \cup\{z=0, r \leq 1 / 2\}$, i.e. the equatorial plane $z=0$.

In the $(\rho, \chi, \varphi)$ coordinates the metric $\stackrel{o}{g}_{a b}$ takes the form

$$
\stackrel{o}{g}_{a b}=\frac{\rho_{a} \rho_{b}}{1-\rho^{2}}+\left(1-\rho^{2}\right) \chi_{a} \chi_{b}+\rho^{2} \varphi_{a} \varphi_{b} .
$$

We now generalize Eq. (7) by setting

$$
g_{a b}=e^{A q}\left[\frac{\rho_{a} \rho_{b}}{1-\rho^{2}}+\left(1-\rho^{2}\right) \chi_{a} \chi_{b}\right]+\rho^{2} \varphi_{a} \varphi_{b}
$$

where $A$ is a constant and $q$ is a smooth function of $\rho^{2}$ with $\left.q\right|_{\rho=0}=0$. Eq. (8) then defines a smooth metric on $S^{3}$ which, again, has a $U(1) \times U(1)$ symmetry with the same properties as above, with the exception that the hemispheres $\chi=$ const. and $\varphi=$ const. are not now metric half-spheres and that maximal tori $\rho=\rho_{0}$ occur whenever

$$
A \rho\left(1-\rho^{2}\right) q^{\prime}+1-2 \rho^{2}=0, \quad \text { where } \quad q^{\prime}=\frac{d q}{d \rho} .
$$

The metrics defined by (8) are the free data we wish to consider. The first step now consists of checking whether $\lambda_{1}(g)>0$. This is known to be true for $A_{1}<A<A_{2}$ [5], where $A_{1}<0, A_{2}>0$ are numbers dependig on $q$, both finite provided $q \not \equiv 0$. In the present case $\lambda_{1}(A)$ is determined by an ODE: by virtue of non-degeneracy of ground states, the corresponding eigenfunction has to share the $U(1) \times U(1)$-symmetry of the problem and is a function merely of $\rho$. (In contrast, in Eq. (2), the original symmetry is broken by one's 
choice of $\Lambda$.) It is known that for $A$ sufficiently close to $A_{1}$ or $A_{2}$, the constant term in $G$, i.e. the ADM mass gets arbitrarily large and the physical metric $g^{\prime}$ develops trapped surfaces (see Ref. [3]).

In the next step we subtract a suitable $G_{l o c}$ from $G$ and try to solve Eq. (3). We can now expand $\phi$ and $\rho_{\lambda}$ in a Fourier series on the square torus $(\chi \in[0,2 \pi], \varphi \in[0,2 \pi])$. Since $L_{g}$ commutes with $\frac{\partial}{\partial \chi}$ and $\frac{\partial}{\partial \varphi}$ this results in an ODE on $\rho \in[0,1]$ for each Fourier mode, supplemented by boundary conditions at $\rho=0$ and $\rho=1$ needed to ensure regularity of $\phi$ on the two axes. By uniqueness [6] an isometry of $g_{a b}$ carries over to $g_{a b}^{\prime}$ iff $\Lambda$ is a fixed point of this isometry. Thus, if $\Lambda$ is in a general position, $g^{\prime}$ has no isometries whatsoever. On the other hand, taking $\Lambda$ to be at $\rho=0, \chi=0$, say, we keep $\frac{\partial}{\partial \varphi}$ as a Killing vector, and the resulting data are a special case of Brill waves [7]. The vector field $\frac{\partial}{\partial \chi}$ survives on $R^{3}=S^{3} \backslash \Lambda$ merely as a conformal vector field which is incomplete along the axis $\rho=0$. There is however the discrete "mirror" isometry $(\rho, \chi, \varphi) \mapsto(\rho, 2 \pi-\chi, \varphi)$, leaving the equator invariant.

We can generalize the above to the case where there are several asymptotic ends. We simply choose a finite number of points $\Lambda_{i}(i=1, \ldots, N)$, solve numerically for the corresponding Green function $G_{i}$ and write

$$
g_{a b}^{\prime}=\left(\sum_{i=1}^{N} c_{i} G_{i}\right)^{4} g_{a b},
$$

where the "source strengths" $c_{i}$ are arbitrary positive numbers. When $A=0$, these are simply the many-black-hole solutions discussed by Brill and Lindquist [8].

If all $\Lambda_{i}$ lie on the two axes, each $G_{i}$ in $G=\sum_{i=1}^{N} c_{i} G_{i}$ corresponds to an axially symmetric (Brill-type) solution. It is perhaps useful to observe that Brill's simple positive-mass proof [7] immediately gives positivity of mass also in this non-axially symmetric, topologically nontrivial situation. Another useful observation, in the case of a general distribution of $\Lambda_{i}$ 's, is that not all of the $G_{i}$ 's have to be computed separately: for any $\Lambda_{1}, \Lambda_{2}$ lying on the same orbit of the isometry group, $G_{2}$ is given in terms of $G_{1}$ by a product of suitable rotations in $\varphi$ and $\chi$. Take e.g. $N=2$ with $\Lambda_{1}$ at $(\rho=0, \chi=0)$ and $\Lambda_{2}$ at $(\rho=0, \chi=\alpha)$. In the special case $\alpha=\pi$, the physical metric $g_{a b}^{\prime}$ has the mirror symmetry $\chi \mapsto 2 \pi-\chi$. 
When $c_{1}=c_{2}$, it has the inversion symmetry $\{\chi\} \cup\{\chi+\pi\} \mapsto\{\alpha-\chi\} \cup\{\alpha-\chi+\pi\}$, leaving the "throat" $\{\chi=\alpha / 2\} \cup\{\alpha / 2+\pi\}$ invariant. Thus the "throat" is totally geodesic with respect to $g_{a b}^{\prime}$, in particular a minimal embedded 2-sphere (a "horizon"). We remark that, when $A=0$, the above discrete symmetries are present irrespective of $c_{1}, c_{2}$ and $\alpha$. Because, then, by combining a homothety with a proper conformal motion coming from the Killing vector $\frac{\partial}{\partial z}$ on $\left(R^{3}, g_{a b}^{\prime}\right)$, we can always arrange for $c_{1}^{\prime}=c_{2}^{\prime}, \alpha^{\prime}=\pi$. The corresponding physical data is of course nothing but a time-symmetric slice of the Kruskal spacetime with mass $m\left(c_{1}, c_{2}, \alpha\right)=2 \sqrt{2} c_{1} c_{2}(1-\cos \alpha)^{-1 / 2}$. When $A \neq 0$ our solutions for $c_{1} \neq c_{2}$ are not inversion symmetric. Placing sources of equal strength at $\rho=0, \alpha_{i}=\frac{2 \pi i}{N}, i=0,1, \ldots, N-1$, we find in an analogous manner that on $R^{3} \backslash \bigcup_{i=1}^{n} \Lambda_{i}$ each asymptotic end is surrounded by a throat. Thus, viewing $\Lambda_{1}$ as "infinity" and the other $\Lambda_{i}$ 's as "black holes" or "particles" we can in particular say that these objects, viewed from infinity, are so close together that, in addition to a horizon for each of them individually, there is one surrounding them all.

Finally we want to place the solutions found here in a more general context. From the conformal-compactification viewpoint adopted here, looking for time-symmetric initial data with symmetries naturally leads to looking for metrics $g$ on a compact 3 -manifold $M$, not necessarily $S^{3}$, with a group $\mathcal{G}$ of conformal symmetries. Suppose $(M, g)$ is not conformally diffeomorphic to $\left(S^{3}, \stackrel{o}{g}\right.$ ) (in which case the one-point conformal decompactification would be data for Minkowski spacetime. Then, by virtue of a theorem due to Obata [9], $\mathcal{G}$ acts by isometries on a metric $g_{1}$ in the conformal class of $g$. Thus, calling $g_{1}$ again $g$, we are left with isometries of $(M, g)$. When $\mathcal{G}$ has 3 -dimensional orbits, it has to be one of the Bianchi types existing on a compact manifold. Whether they too can be usefully employed as background metrics for conformal decompactification, we have not investigated. The next case is the one of 2-dimensional orbits which has been fully classified [10]. Taking $M$ to be $S^{3}$, which is the topologically trivial case in the present context, $\mathcal{G}$ can be $S O(3)$ or $U(1) \times U(1)$. The first case is again conformally, whence physically, trivial. The second case, provided the two Killing vectors can be chosen to be orthogonal, is the polarized Gowdy case, which is conformally diffeomorphic to the one used here. 
If $M$ is chosen to be $S^{2} \times S^{1}$ and $\mathcal{G}=S O(3),(M, g)$ is conformally equivalent to $S^{2} \times S(a)$, the standard $S^{2}$ times the circle of radius $a$. This, in physical terms, corresponds to the Misner wormhole [11]. The case $\mathcal{G}=U(1) \times U(1)$ is a generalization of the Misner wormhole, and is similar to the case studied here.

Finally, when $M \cong T^{3}$, it follows from a fundamental theorem of Schoen and Yau [12] that $\lambda_{1}(g) \leq 0$ for all metrics $g$, so that no positive Green function exists.

Let us summarize: We have presented here a class of initial data for General Relativity which in general have no symmetries whatsoever and for which the constraints are equivalent to an infinite system of uncoupled ODEs. In forthcoming work by one of us (S.H.), the procedure outlined here is carried out for some classes of initial data having the required $U(1) \times U(1)$ conformal symmetry. This will involve a numerical study of the existence question, i.e. the sign of $\lambda_{1}(g)$, and the actual construction of initial data. Also included will be the location of apparent horizons and tests for the isoperimetric inequality of black holes and the hoop conjecture. Since our method is fundamentally different from the one usually employed in Numerical Relativity based on solving PDEs directly on 2 or 3 dimensional grids, we expect our solutions to be useful testbeds for ones obtained by these standard methods. Clearly our method so far makes decisive use of the linearity of the Lichnerowicz equation (2) in the time-symmetric case. In the future we plan to study the nonlinear case of maximal non-time-symmetric initial data having a $U(1) \times U(1)$ symmetry of the background metric and background extrinsic curvature. Whether the lines of thought followed in the present work offer simplifications also for the evolution problem is at present unclear.

This work was supported by Fonds zur Förderung der wissenschaftlichen Forschung, Project No. P09376-PHY. 


\section{REFERENCES}

[1] For a recent study of "asymptotically flat" cylindrical waves, using a weaker notion of asymptotic flatness, see B. K. Berger, P.T. Chrusciel, and V. Moncrief, Ann. Phys. (N.Y.), to be published.

[2] For a recent rigorous discussion see P. T. Chrusciel, Ann. Phys. 202, 100 (1990)

[3] See e.g. R. Beig and N. Ó Murchadha, Phys. Rev. Lett. 66, 2421 (1991) and references cited therein.

[4] P. Garabedian, Partial Differential Equations (Wiley, New York, 1964).

[5] S. Husa, diploma thesis, University of Vienna, 1992.

[6] R. Beig, Class. Quant. Grav. 8, L205 (1991).

[7] D. R. Brill, Ann. Phys. (N.Y.) 7, 466 (1959).

[8] D. R. Brill and R. W. Lindquist, Phys. Rev. 131, 471 (1963).

[9] M. Obata, J. Diff. Geom. 6, 247 (1971); J. Lelong-Ferrand, C.R. Acad. Sci. Paris 269, $583(1969)$.

[10] For a recent account, see Ref. [2].

[11] C. W. Misner, Ann. Phys. (N.Y.) 24, 102 (1963).

[12] R. Schoen and S. T. Yau, Ann. Math. 110, 127 (1979). 